\title{
Molecular genetics of low-grade gliomas: genomic alterations guiding diagnosis and therapeutic intervention. 11th Annual Frye-Halloran Brain Tumor Symposium Meeting Report
}

\author{
Pamela S. Jones, M.D., Gavin P. Dunn, M.D., Ph.D., Fred G. Barker II, M.D., \\ William T. Curry, M.D., Fred H. Hochberg, M.D., and Daniel P. Cahill, M.D., Ph.D. \\ Department of Neurosurgery, Massachusetts General Hospital, Boston, Massachusetts
}

Object. The authors' goal was to review the current understanding of the underlying molecular and genetic mechanisms involved in low-grade glioma development and how these mechanisms can be targets for detection and treatment of the disease and its recurrence.

Methods. On October 4, 2012, the authors convened a meeting of researchers and clinicians across a variety of pertinent medical specialties to review the state of current knowledge on molecular genetic mechanisms of low-grade gliomas and to identify areas for further research and drug development.

Results. The meeting consisted of 3 scientific sessions ranging from neuropathology of IDH1 mutations; CIC, $A T R X$, and FUBPI mutations in oligodendrogliomas and astrocytomas; and IDHI mutations as therapeutic targets. Sessions consisted of a total of 10 talks by international leaders in low-grade glioma research, mutant IDHI biology and its application in glioma research, and treatment.

Conclusions. The recent discovery of recurrent gene mutations in low-grade glioma has increased the understanding of the molecular mechanisms involved in a host of biological activities related to low-grade gliomas. Understanding the role these genetic alterations play in brain cancer initiation and progression will help lead to the development of novel treatment modalities than can be personalized to each patient, thereby helping transform this now often-fatal malignancy into a chronic or even curable disease. (http://thejns.org/doi/abs/10.3171/2012.12.FOCUS12349)

KeY Words • glioma • meeting report • IDH

$\mathrm{T}$ 2012 Frye-Halloran Symposium took place at the Massachusetts General Hospital on October 4, bringing together international experts to address the molecular revolution in the diagnosis and classification of low-grade gliomas. The symposium is an annual event that aims to facilitate the translation of promising innovations from the laboratory to the clinic. Noteworthy past symposia have focused on such topics as the introduction of microdialysis for real-time in situ measurement of activated drugs, the identification of novel MRI findings to track the effects of angiogenesis inhibition, the development of therapies based on viral vectors and cell-based immunizations, and racial diversity in glioma patient populations.

Gliomas represent the most common primary brain tumor, and subtypes occur along a spectrum of aggres-

\footnotetext{
Abbreviations used in this paper: MGMT $=$ O-6-methylguanineDNA methyltransferase; PARP = poly-adenosine diphosphate-ribose polymerase; $\mathrm{PI} 3 \mathrm{~K}$ = phosphatidylinositol 3-kinase.
}

siveness and therapeutic responsiveness. The WHO grading of human brain tumors provides a basis for predicting the clinical behavior of the respective neoplasm by including information beyond a strictly histological grading. ${ }^{16}$ Greater understanding of the molecular genomics of gliomas is allowing a refinement of traditional classification between low- and high-grade gliomas.

Over the past 4 years, several recurring mutations have been identified in low-grade gliomas that, together, have transformed our understanding of this disease. Recently identified gene mutations include those found in the family of isocitrate dehydrogenase (IDH) genes, enzymes involved in citrate metabolism. The IDHI mutation was first reported in 2008, when it was discovered in $12 \%$ of sequenced glioblastoma genomes. ${ }^{21}$ Further research has shown that $I D H$ gene mutations are found in a substantial majority of lower- and intermediate-grade gliomas and are also highly prevalent in secondary glioblastomas that arise from lower-grade tumors. ${ }^{12}$ It has also been associated with longer survival across all grades of 
glioma in which it is found. ${ }^{1,25}$ The emerging clinical phenotype of the IDHI mutation is that of a younger patient ( $<44$ years), with frontal lobe tumor location, and a significant component of non-contrast enhancing tumor on imaging. ${ }^{15}$ Low-grade gliomas can be further subdivided into groups characterized by astrocytic or oligodendroglial histology. Recent findings have identified genetic alterations associated with these subgroups as well.

With these discoveries of specific recurrent mutations, we are now on the cusp of translating these molecular discoveries into meaningful clinical strategies for patient care. This symposium was convened to set the stage for upcoming clinical trials and opportunities for collaboration in the study and care of patients with lowgrade glioma.

The meeting was divided into 3 sessions: 1) Neuropathology of IDH1 Mutations; 2) Mutations in LowGrade Gliomas: $A T R X, T P 53, C I C$, and FUBPI; and 3) IDH1 Mutations as Therapeutic Targets. The audience included clinicians and laboratory scientists in the fields of neurooncology, neurosurgery, neuropathology, radiation oncology, neuroradiology, pediatrics, nursing, and other specialties involved in the research, diagnosis, care, and treatment of patients with low-grade gliomas. This article summarizes the highlights of each of the 10 presentations. Readers are referred to the conference website (http://fh2012.neurosuite.org/) and the program brochure (http://www.dfhcc.harvard.edu/uploads/media/2012_ Frye_Halloran.pdf) for further details.

\section{Session 1: Neuropathology of IDH1 Mutations}

The meeting opened with a discussion of neuropathology by David Louis, M.D. (Massachusetts General Hospital, Boston, Massachusetts), who reviewed the evolving tools by which we may define low-grade gliomas. The WHO 2007 criteria were the first to include molecular and genomic analyses in brain tumor categorization, specifically recognizing the allelic losses of $1 \mathrm{p}$ and $19 \mathrm{q}$ present in $50 \%-70 \%$ of WHO Grade II and III oligodendrogliomas. ${ }^{16}$ Presence of this deletion has important diagnostic and therapeutic implications, as studies have shown that loss of $1 p$ or loss of both $1 p$ and $19 q$ is a statistically significant predictor of good response to chemotherapeutic combination of procarbazine, lomustine, and vincristine (termed $\mathrm{PCV}) .{ }^{4}$ Conversely, presence of $C D K N 2 \mathrm{~A}$ gene deletions and ring enhancement of the tumor on contrasted imaging both portend significantly worse prognosis and poor response of oligodendrogliomas to PCV. This highlights perhaps 2 tumor subtypes of oligodendrogliomas that can be distinguished almost solely on a genetic basis. With rapid advances in molecular genomics, one could imagine a future in which a molecular diagnosis replaces the traditional pathological diagnosis, so that a tumor is labeled a " $B R A F$ mutant" rather than a pilocytic astrocytoma, ganglioglioma, or pleomorphic xanthoastrocytoma. However, this scenario is currently unlikely, as it seems there is a blend of genetic variations that contributes to unique tumor entities despite their distinct histological phenotypes. Further research is needed to identify the relationships between multiple genetic mutations and the disease phenotype. In the meantime, genetic markers will continue to be powerful tools in guiding diagnosis, prognosis, and therapy.

Andreas von Deimling, M.D. (University of Heidelberg, Germany), presented his laboratory's work on establishing the frequency, distribution, and significance of IDH mutations in gliomas and argued that knowledge of mutant status has allowed us to make important advances in determining the diagnosis and prognosis of gliomas. In analysis of $I D H 1$ and $I D H 2$ mutations in brain tumors, ${ }^{12}$ the highest frequencies of $I D H 1$ mutations were found in diffuse astrocytomas (WHO Grade II, 72.7\%), oligodendrogliomas (WHO Grade II, 82.0\%), and oligoastrocytomas (WHO Grade II, 78\%). IDH2 mutations were highest in oligodendrogliomas (WHO Grade II, 4.7\%), anaplastic oligodendrogliomas (WHO Grade III, 5.2\%), and anaplastic oligoastrocytoma (WHO Grade III, 6.2\%). Overall, IDH1 mutation of R132C showed the strongest association with astrocytomas; conversely, IDH2 mutations were strongly associated with oligodendroglial tumors, and patients with astrocytomas and IDHI mutations were on average 6 years younger than those without the mutation. A monoclonal antibody recognizing the IDHI $\mathrm{R} 132 \mathrm{H}$ mutation in formalin-fixed paraffin-embedded tumor specimens has demonstrated high specificity and sensitivity, ${ }^{6}$ and as a result has greatly facilitated rapid determination of $\mathrm{IDH} 1$ status in tumor classification. ${ }^{5} \mathrm{De}-$ termination of $I D H 1$ status has become especially important given evidence that $I D H 1$ status is perhaps the single most prominent prognostic factor for overall survival after histopathological grading. Sequencing of IDHI codon 132 in 382 patients with anaplastic astrocytoma and glioblastoma from the NOA-04 trial and from a prospective translational cohort study of the German Glioma Network showed that $I D H I$ status correlated with prognosis more than age, histological diagnosis, or MGMT methylation status. As such, the combination of histological diagnosis with genomic features could be ranked from most favorable to least favorable: 1) anaplastic astrocytoma with IDHI mutation, 2) glioblastoma with IDHI mutation, 3) anaplastic astrocytoma without IDHI mutation, and 4) glioblastoma without IDHI mutation. ${ }^{11}$ These findings suggest a potential guide for recategorization of WHO tumor classification to more accurately delineate tumor types in the age of molecular genomics.

\section{Session 2: Mutations in Low-Grade Gliomas: $C I C, A T R X$, and FUBP1}

Daniel Cahill, M.D., Ph.D. (Massachusetts General Hospital), discussed the potential for clinical impact of IDHI status on driving tumor classification and therapy. Due to the significant prognostic differences between patients harboring tumors with and without the IDHIR132H mutation, we can begin to consider a tumor's IDH1 mutant status as having distinct biological properties that belong to a separate molecular etiological group altogether, and, as such, merit a uniquely tailored treatment strategy. The value of maximum resection in gliomas has been controversial, and there exists evidence that the prognostic significance of resection may be biased due to gross-total resections occurring more frequently 


\section{Molecular genetics of gliomas: Frye-Halloran Meeting Report}

in younger patients and in noneloquent locations. ${ }^{22}$ Interestingly, there is also a predominant occurrence of IDHI mutations in younger patients and tumor locations in the frontal lobe. Overall, these correlations suggest that measures of resection may be difficult to isolate from other confounding molecular prognostic factors, without explicit stratification. In a study of 407 patients with malignant astrocytomas (157 anaplastic astrocytomas and 250 glioblastomas), the measures of resection associated with longer survival differed between separately analyzed IDH1 wild-type and mutant tumors. Controlling for age, Karnofsky performance score, tumor location, and tumor grade, patients with IDHI wild-type tumors showed extended survival when all enhancing disease was resected, but postoperative residual nonenhancing disease was not associated with survival. ${ }^{3}$ Conversely, in patients with IDH1 mutant tumors, both residual enhancing and nonenhancing postoperative tumor burden were associated with worse survival. These data suggest an additional survival benefit that appears to be associated with reducing nonenhancing tumor burden in $I D H 1$ mutant tumors. Thus, determining $I D H 1$ genotype may serve to uniquely guide surgical planning, which may include more aggressive resection in patients with mutant tumors.

Stephen Yip, M.D., Ph.D. (University of British Columbia, Vancouver, Canada), presented his laboratory's data on the recently discovered genetic aberration of the CIC gene on $19 \mathrm{q}$ of oligodendrogliomas. The CIC gene is located on 19q13.2 and encodes a transcriptional repressor that is a mammalian homolog of the Drosophila Capicua that plays a crucial role in development via signaling downstream of the RAS/MAPK pathway. Normal mammalian Capicua is found frequently in the brain, and CIC mutations have previously been identified in spinocerebellar ataxia I (SCAI) and Ewing sarcomas. In addition to the $I D H I$ and $I D H 2$ mutations and $1 \mathrm{p} / 19 \mathrm{q}$ loss that have already been strongly linked to oligodendrogliomas, the $C I C$ gene was found to be mutated in $20(69 \%)$ of 29 oligodendrogliomas with $1 \mathrm{p} 19 \mathrm{q}$ codeletion that were studied with whole exome sequencing. ${ }^{26}$ In contrast, deep sequencing of astrocytomas and oligodendrogliomas without $1 \mathrm{p} / 19 \mathrm{q}$ deletion had rare loss of $C I C$ (only 1 [2\%] of 60). When examining 204 tumor samples, the CIC mutation was closely associated with classic oligodendroglioma histology and also with $I D H 1 / 2$ mutations and loss of $1 \mathrm{p} / 19 \mathrm{q}$. After controlling for $1 \mathrm{p} / 19 \mathrm{q}$ status, there was a trend of association between CIC mutation and high-grade status, but no association with overall or progression-free survival. No $C I C$ mutations were identified in 4 normal brain tissue samples. Since the prototypical oligodendroglioma involves abnormalities of $C I C, I D H$, and $1 \mathrm{p} / 19 \mathrm{q}$, this suggests that all 3 are involved in the underlying biology of this tumor class. Further research to elucidate the complex relationship and mechanisms of these genetic abnormalities is necessary to understand tumor development and help guide future therapy.

Chetan Bettegowda, M.D., Ph.D. (Johns Hopkins, Baltimore, Maryland), further discussed the CIC mutation and his recent work to genetically profile anaplastic oligodendrogliomas. Beyond the hallmark genetic markers of $I D H$ mutation and $1 \mathrm{p} / 19 \mathrm{q}$ codeletions, whole exome sequencing of 7 anaplastic oligodendrogliomas also revealed common mutations of NOTCH1 (2 tumors), PIK3CA (3 tumors), CIC (6 tumors), and FUBPI (2 tumors). ${ }^{2}$ This high rate of $C I C$ mutation was validated by analyzing 27 additional tumors, and, in total, CIC mutations were identified in 18 of 34 samples. While no additional $\mathrm{NOTCH} 1$ mutations were found in the validation set, FUBP1 alterations were ultimately found in 5 of 34 samples. The gene for the far-upstream element binding protein $(F U B P)$ is located on $1 \mathrm{p}$ and encodes a protein that binds to single-stranded DNA, with a known target site within the far-upstream element of the $M Y C$ oncogene. All mutations identified were found to inactivate FUBP1. Of the 34 studied oligodendroglial tumors, 23 had either $C I C$ or FUBPI mutations, and all of the $C I C$ mutations occurred in tumors that also had loss of $19 \mathrm{q}$. Additional research into these oligodendroglioma mutations will provide insight into tumor pathogenesis and diagnosis and may also inform tailored therapies. In particular, discussion centered on the yet-unclear relationship between chemosensitivity observed in the clinical studies of 1p/19q-deleted oligodendroglioma by Cairncross et al. ${ }^{4}$ and others and the hypothesized mechanisms of tumor promotion of these molecular genetic alterations.

Zachary Reitman, Ph.D. (Duke University, Durham, North Carolina), presented data on the ATRX mutation recently found in genetic profiling of 363 gliomas. The alpha-thalassemia/mental retardation syndrome X-linked $(A T R X)$ was recently identified in $7 \%$ of sequenced adult glioblastomas as well as in pancreatic neuroendocrine tumors, ${ }^{13}$ but had not yet been examined in gliomas of all grades. It is an adenosine triphosphate-dependent helicase that maintains telomere homeostasis by incorporating histone variant H3.3 into telomeric chromatin. The mutations of gliomas cause loss of protein expression, whereby the alternative lengthening of telomeres (ALT) pathway is activated, a common pathway used by cancerous cells. Mutations of ATRX were found in 93 of the 363 brain tumor samples examined and occurred across 10 tumor subtypes..$^{14}$ Most frequent mutations were found in Grade II diffuse astrocytomas $(67 \%, \mathrm{n}=15)$, Grade III anaplastic astrocytomas $(73 \%, \mathrm{n}=40)$, secondary glioblastomas $(57 \%, \mathrm{n}=14)$, and oligoastrocytomas $(68 \%$, $\mathrm{n}=40)$. Interestingly, there were no ATRX mutations in Grade I pilocytic astrocytomas, and there was a much lower rate in primary (or de novo) glioblastoma (4\%, $\mathrm{n}=$ 94) when compared with secondary glioblastoma. Furthermore, $A T R X$ alterations in the gliomas were found to co-occur with TP53 and IDH alterations, and this constellation of mutations was significantly associated with ALT expression. Of note, $A T R X$ alteration and 1p/19q loss only co-occurred in a single case. As for prognostic analysis, it was intriguing to note that survival was significantly longer in patients with Grade II-IV gliomas harboring both IDH and ATRX mutations (median 51 months) compared with $I D H$ mutation alone (median 28 months). Further analysis of $C I C$ and $F U B P I$ alterations showed that tumor types with $I D H$ mutation, combined with $1 \mathrm{p} / 19 \mathrm{q}$ loss, CIC mutation, or FUBPI mutation, had significantly improved survival (median 96 months). Overall, while the molecular mechanisms have yet to be fully character- 
ized, these data support the theory that 2 core subclasses of low-grade gliomas may be differentiated by unique genetic signatures, whereby a combination of IDH and ATRX mutations yield a different phenotype (astrocytic) than a combination of $I D H$ and $1 \mathrm{p} / 19 \mathrm{q}, C I C$, or $F U B P I$ mutations (oligodendroglial). Further exploration into the intricate molecular landscape of gliomas will likely contribute to more distinct tumor subtype classifications.

\section{General Session: Mutant IDH Enzymes and Cancer Biology}

Ross Levine, M.D. (Memorial Sloan-Kettering Cancer Center, New York, New York), led a general audience through Grand Rounds on how IDH mutations cause leukemia via aberrant epigenetic programming from DNA hypermethylation and reduced enzyme activity. Similar to gliomas, acute myeloid leukemia is one of the cancers bearing $I D H 1$ and $I D H 2$ mutant phenotypes, albeit at lower incidences..$^{10,18}$ Moreover, AML still carries a poor overall survival under current standard treatments. Just as the IDHI mutant form of glioma is associated with improved survival, AML survival can also be stratified based on mutational profiling, where $I D H 2$ mutant AML predicts favorable outcome. The mechanisms involved in IDHI mutant leukemic biology have yet to be fully elucidated. Mutational and epigenetic profiling of a large cohort of patients have demonstrated that $I D H 1$ and $I D H 2$ mutant AMLs display global DNA hypermethylation and a specific hypermethylation signature. ${ }^{10}$ Follow-up studies show that $I D H$ mutant gliomas also have a distinct hypermethylation signature, known as $\mathrm{CpG}$ island methylation profile (CIMP). ${ }^{20,23}$ Additionally, it is believed that the 2-hydroxyglutarate (2-HG) produced by IDH mutant enzymes can accumulate in cells and prevent histone demethylation that is required for lineage-specific progenitor cells to differentiate into terminally differentiated cells. ${ }^{17,24}$ These studies demonstrate that the neomorphic enzymatic function of the IDH proteins likely affects gene expression programs, at least in part, by altering the epigenome via methylation changes in both DNA and core histone proteins. The novel enzyme function of $I D H$ mutants therefore represents a promising potential drug target.

\section{Session 3: IDH1 Mutations as Therapeutic Targets}

David Schenkein, M.D. (Agios Pharmaceuticals, Cambridge, Massachusetts), began the final session by sharing what lies on the horizon for $I D H$-related therapeutic development. There is a view that tumor-associated alterations in metabolic pathways lead to a phenoytpe under which tumor cells can survive and grow. To this end, targeting metabolic enzymes to treat cancer offers a promising area of oncology research. This idea has gained ground with the study of "metabolomics," which involves rapid, high-throughput characterization of the small molecule metabolites found in tissues, biofluids, or organisms in general. Development of a "metabolome" for different tumor types offers the possibility of uncovering many novel therapeutic targets. For instance, the
IDHI-mutant metabolome is associated with high levels of 2-HG in cells expressing IDHI-R132H, and this oncometabolite is detectable in brain tumor samples, in CSF, and on MR spectroscopy. Thus, levels of 2-HG could be measured during treatment in the event that mutant $I D H I$ enzymes could be inhibited pharmacologically. Furthermore, studies have indicated that high levels of 2-HG can drive epigenetic changes that inhibit cell differentiation and contribute to cancer. Thus, engineering molecules to inactivate the $I D H$ mutant enzymes that produce 2-HG will in theory antagonize these downstream effects, leading to tumorigenesis. ${ }^{8}{ }^{8} 92-\mathrm{HG}$ levels can indicate the effectiveness of such therapies. Agios Pharmaceuticals has developed potent and orally available selective inhibitors of both $I D H I$ and $I D H 2$ mutant enzymes. Preliminary studies of in vivo tumor models have shown they are capable of lowering $2 \mathrm{HG}$ levels by greater than $90 \%$ and reversed the altered methylation profiles of the $I D H$ mutant cells. The biology of mutant IDH proteins is one of a few examples of metabolic dysregulation affecting epigenetics. If clinical pharmacological development continues successfully, we may expect $I D H$ mutant-selective therapies to become available soon.

Lewis Cantley, Ph.D. (Beth Israel, Boston, Massachusetts), discussed how carefully designed clinical trials using drug combinations may offer the key to developing successful cancer treatments. As previously mentioned, numerous consistent genetic mutations are known to occur within gliomas, including alterations in IDH, TP53, $P T E N, N F 1$, and $E G F R$, to name a few. Given that tumor phenotypes are the result of the interplay among multiple aberrant molecular pathways, successful glioma treatment likely resides in the inhibition or modulation of more than one such pathway. Thus, as we explore therapeutic targets for $I D H$ mutations in gliomas, coclinical trials of 2 or more therapies may be strategically advantageous. One illustration of combination molecular drug therapy development is that of a BRCAl-mutant mouse model treated with a PI3K inhibitor and a PARP inhibitor. The PI3K inhibitor, BKM120, alters pathways associated with cell proliferation and glucose metabolism. The PARP inhibitor olaparib prevents repair of DNA breaks, which preferentially leads to death in rapidly replicating tumor cells. In BRCA1-mutant mouse models, PI3K inhibition slowed tumor growth. However, the drug caused activation of PARP to help repair DNA damage. Addition of a PARP inhibitor further promoted DNA destruction and eventual cancer cell death, creating an unexpected synergistic effect. This approach to combination therapeutics may prove successful in glioma treatment as well. For instance, recent studies have indicated that there is evidence that altered glucose metabolism can affect prognosis for gliomas..$^{19}$ This may warrant exploration of the synergistic effects of current standard therapies with a PIK3 inhibitor or another molecular target in the glucose metabolism pathway. Ultimately, it may be important to simultaneously target different mechanisms in gliomagenesis.

Linda Liau, M.D., Ph.D. (UCLA, Los Angeles, California), gave the final presentation of the symposium by presenting data regarding the clinical implications of IDH gene mutations on glioma therapy. In support of the 


\section{Molecular genetics of gliomas: Frye-Halloran Meeting Report}

differing imaging characteristics and prognoses between IDH1 mutant and wild-type gliomas, there is recent evidence to suggest that $I D H 1$ mutant glioblastoma evolves from a cell of origin distinct from that of glioblastomas without $I D H 1$ mutation. ${ }^{15}$ One of the key distinctions is that gliomas with the IDHI mutation have been found to harbor a distinct $\mathrm{CpG}$ island hypermethylation profile $(C I M P)$, which is absent in $I D H 1$ wild-type gliomas. ${ }^{20} \mathrm{It}$ is theorized that this hypermethylation profile contributes to glioma formation by silencing tumor suppressor genes. A recent study looked at candidate tumor suppressor genes by performing bisulfate sequencing on $198 \mathrm{IDH} / / 2 \mathrm{mu}-$ tant and IDH wild-type gliomas (Grades III and IV). One particular gene that was found to be hypermethylated was that of $R B P 1$, which is involved in retinoic acid metabolism. Nearly all the IDH1 mutant (76/79) and IDH2 mutant (3/3) tumors demonstrated RBPI hypermethylation, versus none of the $119 \mathrm{IDH}$ wild-type tumors. In further analysis of the glioblastoma cohort, the patients with $R B P 1$ hypermethylation $(\mathrm{n}=22)$ had a statistically significantly higher median overall survival compared with RBPI unmethylated patients $(\mathrm{n}=102)$ of 36.9 months versus 20.3 months, respectively. This was expected due to the close association of $R B P 1$ hypermethylation and $I D H$ mutation status. When controlling for clinical variables of age, sex, performance status, and extent of resection, analysis showed that either IDHI/2 mutation or $R B P 1$ methylation status is an independent predictor of overall survival. ${ }^{7}$ MGMT promoter methylation status has already served as a valuable clinical predictor of response to standard-of-care glioblastoma therapy. As such, further research of the hypermethylation of $R B P 1$ and other genes may provide key insight for both identifying targets of future glioma therapy and determining prognosis.

\section{Conclusions}

The data presented at the 11th Annual Frye-Halloran Symposium highlighted the rapidly moving field of lowgrade glioma molecular genetics. Recent discoveries about the underlying genetic profiles of low-grade gliomas, following the seminal discovery of IDH gene mutations, have led to a revolution in our understanding of the genetic alterations that are mechanistically involved in gliomagenesis. Identification of recurrent mutations in novel genes such as $C I C, F U B P 1$, and $A T R X$ certainly justifies the investment in large-scale exome-wide sequencing studies that have driven these discoveries. Lowgrade gliomas, specifically, pose a unique challenge in classification, given the infiltrative nature of the tumor cells and the heterogeneity of genotypes and phenotypes. In a relatively brief period of time, there are now multiple objective mutational alterations identified that will allow for the reproducible biological subclassification of these tumors. It will be interesting to observe how rapidly these molecular features become integrated into standard diagnostic categorization and in what capacity. For instance, there now exists potential for establishing diagnostic biomarkers, whereby a tumor's genetic alterations can be identified from serum or CSF rather than a surgical procedure. Importantly, these genetic alterations are potential targets themselves, and the possibility that these mutations or nodes in their associated biology can be therapeutically targeted in the near future offers substantial hope for improvement in the survival of patients with these cancers.

\section{Disclosure}

The Carolyn Frye-Halloran Brain Tumor Therapy Program sponsored the symposium.

Author contributions to the study and manuscript preparation include the following. Conception and design: Cahill, Jones, Barker, Curry. Acquisition of data: Jones. Analysis and interpretation of data: Jones. Drafting the article: Jones. Critically revising the article: all authors. Reviewed submitted version of manuscript: all authors.

\section{References}

1. Balss J, Meyer J, Mueller W, Korshunov A, Hartmann C, von Deimling A: Analysis of the IDH1 codon 132 mutation in brain tumors. Acta Neuropathol 116:597-602, 2008

2. Bettegowda C, Agrawal N, Jiao Y, Sausen M, Wood LD, Hruban RH, et al: Mutations in CIC and FUBP1 contribute to human oligodendroglioma. Science 333:1453-1455, 2011

3. Cahill DP, Beiko J, Suki D, Prabhu SS, Weinberg J, Lang FF, et al: IDH1 status and survival benefit from surgical resection of enhancing and nonenhancing tumor in malignant astrocytomas. J Clin Oncol Suppl 30:abstr 2019, 2012 (Abstract)

4. Cairncross JG, Ueki K, Zlatescu MC, Lisle DK, Finkelstein DM, Hammond RR, et al: Specific genetic predictors of chemotherapeutic response and survival in patients with anaplastic oligodendrogliomas. J Natl Cancer Inst 90:1473-1479, 1998

5. Capper D, Sahm F, Hartmann C, Meyermann R, von Deimling A, Schittenhelm J: Application of mutant IDH1 antibody to differentiate diffuse glioma from nonneoplastic central nervous system lesions and therapy-induced changes. Am J Surg Pathol 34:1199-1204, 2010

6. Capper D, Weissert S, Balss J, Habel A, Meyer J, Jäger D, et al: Characterization of R132H mutation-specific IDH1 antibody binding in brain tumors. Brain Pathol 20:245-254, 2010

7. Chou AP, Chowdhury R, Li S, Chen W, Kim AJ, Piccioni DE, et al: Identification of retinol binding protein 1 promoter hypermethylation in isocitrate dehydrogenase 1 and 2 mutant gliomas. J Natl Cancer Inst 104:1458-1469, 2012

8. Dang L, Jin S, Su SM: IDH mutations in glioma and acute myeloid leukemia. Trends Mol Med 16:387-397, 2010

9. Dang L, White DW, Gross S, Bennett BD, Bittinger MA, Driggers EM, et al: Cancer-associated IDH1 mutations produce 2-hydroxyglutarate. Nature 462:739-744, 2009

10. Figueroa ME, Abdel-Wahab O, Lu C, Ward PS, Patel J, Shih A, et al: Leukemic IDH1 and IDH2 mutations result in a hypermethylation phenotype, disrupt TET2 function, and impair hematopoietic differentiation. Cancer Cell 18:553-567, 2010

11. Hartmann C, Hentschel B, Wick W, Capper D, Felsberg J, Simon M, et al: Patients with IDH1 wild type anaplastic astrocytomas exhibit worse prognosis than IDH1-mutated glioblastomas, and IDH1 mutation status accounts for the unfavorable prognostic effect of higher age: implications for classification of gliomas. Acta Neuropathol 120:707-718, 2010

12. Hartmann C, Meyer J, Balss J, Capper D, Mueller W, Christians A, et al: Type and frequency of IDH1 and IDH2 mutations are related to astrocytic and oligodendroglial differentiation and age: a study of 1,010 diffuse gliomas. Acta Neuropathol 118:469-474, 2009

13. Heaphy CM, de Wilde RF, Jiao Y, Klein AP, Edil BH, Shi C, et al: Altered telomeres in tumors with ATRX and DAXX mutations. Science 333:425, 2011

14. Jiao Y, Killela PJ, Reitman ZJ, Rasheed AB, Heaphy CM, de 
Wilde RF, et al: Frequent ATRX, CIC, and FUBP1 mutations refine the classification of malignant gliomas. Oncotarget 3: 709-722, 2012

15. Lai A, Kharbanda S, Pope WB, Tran A, Solis OE, Peale F, et al: Evidence for sequenced molecular evolution of IDH1 mutant glioblastoma from a distinct cell of origin. J Clin Oncol 29:4482-4490, 2011

16. Louis DN, Ohgaki H, Wiestler OD, Cavenee WK, Burger PC, Jouvet A, et al: The 2007 WHO classification of tumours of the central nervous system. Acta Neuropathol 114:97-109, 2007

17. Lu C, Ward PS, Kapoor GS, Rohle D, Turcan S, Abdel-Wahab $\mathrm{O}$, et al: IDH mutation impairs histone demethylation and results in a block to cell differentiation. Nature 483:474-478, 2012

18. Mardis ER, Ding L, Dooling DJ, Larson DE, McLellan MD, Chen $\mathrm{K}$, et al: Recurring mutations found by sequencing an acute myeloid leukemia genome. N Engl J Med 361:10581066, 2009

19. McGirt MJ, Chaichana KL, Gathinji M, Attenello F, Than K, Ruiz AJ, et al: Persistent outpatient hyperglycemia is independently associated with decreased survival after primary resection of malignant brain astrocytomas. Neurosurgery 63:286-291, 2008

20. Noushmehr H, Weisenberger DJ, Diefes K, Phillips HS, Pujara $\mathrm{K}$, Berman BP, et al: Identification of a $\mathrm{CpG}$ island methylator phenotype that defines a distinct subgroup of glioma. Cancer Cell 17:510-522, 2010

21. Parsons DW, Jones S, Zhang X, Lin JC, Leary RJ, Angenendt $\mathrm{P}$, et al: An integrated genomic analysis of human glioblastoma multiforme. Science 321:1807-1812, 2008
22. Stummer W, Reulen HJ, Meinel T, Pichlmeier U, Schumacher $\mathrm{W}$, Tonn JC, et al: Extent of resection and survival in glioblastoma multiforme: identification of and adjustment for bias. Neurosurgery 62:564-576, 2008

23. Turcan S, Rohle D, Goenka A, Walsh LA, Fang F, Yilmaz E, et al: IDH1 mutation is sufficient to establish the glioma hypermethylator phenotype. Nature 483:479-483, 2012

24. Xu W, Yang H, Liu Y, Yang Y, Wang P, Kim SH, et al: Oncometabolite 2-hydroxyglutarate is a competitive inhibitor of $\alpha$-ketoglutarate-dependent dioxygenases. Cancer Cell 19:1730, 2011

25. Yan H, Parsons DW, Jin G, McLendon R, Rasheed BA, Yuan W, et al: IDH1 and IDH2 mutations in gliomas. N Engl J Med 360:765-773, 2009

26. Yip S, Butterfield YS, Morozova O, Chittaranjan S, Blough $\mathrm{MD}$, An J, et al: Concurrent CIC mutations, IDH mutations, and $1 \mathrm{p} / 19 \mathrm{q}$ loss distinguish oligodendrogliomas from other cancers. J Pathol 226:7-16, 2012

Manuscript submitted October 19, 2012.

Accepted December 10, 2012.

Please include this information when citing this paper: DOI: 10.3171/2012.12.FOCUS12349.

Address correspondence to: Daniel P. Cahill, M.D., Ph.D., Department of Neurosurgery, Massachusetts General Hospital, 55 Fruit Street, YAW 9E-9026, Boston, Massachusetts 02114. email: dcahill@partners.org. 